\title{
Auf die frühen Phasen kommt es an!
}

Die Produktgestaltung ist ein bedeutendes umweltpolitisches Instrument in Unternehmen. Durch sie werden die mit den Produkten verbundenen Ressourcenbeanspruchungen und Umweltbelastungen festgelegt. Das Ziel ökologischer Produktgestaltung ist es, negative Umwelteinwirkungen, die ein Produkt entlang der Produktlinie verursacht, bereits in der Produktentwicklung zu vermeiden oder zu verringern. Wie das Unternehmensbeispiel AEG zeigt, lassen sich hier noch viele Potenziale erschließen.

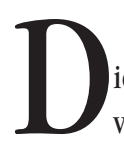
Produktostaltung in Unternehmen verläuft in verschiedenen Arbeitsphasen, die sich grob entsprechend Tabelle 1 charakterisieren lassen. Die Phasen werden tendenziell nacheinander durchlaufen, wobei es zu ständigen Rückkopplungen zwischen den einzelnen Phasen kommt. Konzepte für ökologische Produktgestaltung sollten an diesem bewährten Vorgehen der Unternehmen anknüpfen und es um ökologische Fragestellungen ergänzen.

Von ökologischer Produktgestaltung kann dann gesprochen werden, wenn in allen Gestaltungsphasen ökologische Aspekte berücksichtigt werden und hierbei das Ziel verfolgt wird, Produkte entlang ihrer gesamten Produktlinie ökologisch zu optimieren. Für proaktiven Umweltschutz ist es erforderlich, den frühen Entwicklungsphasen große Bedeutung beizumessen. Er kann nur wirksam werden, wenn die zukünftigen Entwicklungen der Rahmenbedingungen zu Beginn des Gestaltungsprozesses systematisch erfasst und Gestaltungsziele hieran ausgerichtet werden.

Eine Untersuchung der Produktgestaltung der AEG Hausgeräte $\mathrm{GmbH}$ - ein Unternehmen mit einer mehr als zwanzigährigen Tradition in öko- wie ökologische Aspekte bislang in der Produktgestaltung berïcksichtigt werden und wie ihre Einbeziehung intensiviert werden kann (1). Die Untersuchung bestand in der Auswertung betrieblicher Vorschriften zur Produktgestaltung sowie aus Interviews mit verschiedenen innerbetrieblichen AkteurInnen, die am Gestaltungsprozess beteiligt sind.

Die Entwicklung ökologischer Produkte ist erklärtes Ziel der AEG. In der Umweltstrategie des Unternehmens heißt es: „Wir werden ökologisch vernünftige Produkte aktiv fördern, um mitzuhelfen, den Markt hierfür weiter zu entwickeln. Ökologie bedeutet für uns, die Umwelt durch unsere Produkte so wenig wie möglich zu belasten. Umweltschutz beziehen wir nicht nur auf die Entstehung, Nutzung und Verwertung unserer Produkte, sondern auch auf alle Abläufe und Tätigkeiten des Unternehmens." (2) Die Bedeutung des Umweltschutzes in der Produktentwicklung spiegelt sich in einer Vielzahl von Werknormen wider. Neben einer Werknorm zum Gestaltungsablauf gibt es mehrere Vorschriften, die einzelne Aspekte ökologischer Produktgestaltung (wie etwa Stoffeinsatz und recyclinggerechte Konstruktion) behandeln.

Trotz dieser guten Voraussetzungen und der

\begin{tabular}{|c|c|}
\hline Phase & Ergebnis \\
\hline Strategieplanung & $\begin{array}{l}\text { Produktstrategie: relevante Entwicklungsziele für die nächsten Jahre sind festgelegt } \\
\text { unterschiedliche Produktanforderungen sind gewichtet }\end{array}$ \\
\hline Initialphase & relevante zu befriedigende Kundenbedürfnisse sind festgelegt \\
\hline Ideenfindung & mögliche Produkt- und Dienstleistungsideen zur Erfüllung der Bedürfnisse sind gesammelt \\
\hline Auswahl & eine oder mehrere Ideen sind zur Realisierung ausgewählt \\
\hline Umsetzung & realisiertes Produkt ist in den Markt eingeführt \\
\hline Evaluation & $\begin{array}{l}\text { aus der Überprüfung des Prozesses und Beobachtung des Produktes sind neue } \\
\text { Produktideen abgeleitet }\end{array}$ \\
\hline
\end{tabular}

logischen Produktinnovationen - hat ermittelt, zahl- reichen ökologischen Erfolge in der Produktgestaltung zeigte die Untersuchung eines konkreten Entwicklungsbeispieles Optimierungsansätze. Im betrachteten Beispiel Wäschetrockner mit Wärmepumpe wurden ökologische Aspekte bisher vor allem in der Umsetzungsphase und weniger in den frühen Entwicklungsphasen berücksichtigt. Zudem wurde das entwickelte Produkt fast ausschließlich bezüglich des Energieverbrauches in der Nutzung optimiert. In diesem Bereich konnte eine erhebliche Reduktion erreicht werden. Die Fallstudie half, eine Reihe von Hemmnissen zu identifizieren, die einer umfassenden ökologischen Produktgestaltung entgegenstanden.

Neben externen Hemmnissen oder schwierigen Rahmenbedingungen (wie etwa Kaufkraft der KundInnen, geringer Energiepreis) gibt es auch eine Reihe innerbetrieblicher Hemmnisse. Diese lassen sich im wesentlichen auf die folgenden vier Ursachen zurückführen, aus denen sich gleichzeitig Ansätze zu ihrer Überwindung ergeben.

\section{(Zu) Technischer Schwerpunkt in der Produktgestaltung}

Die Hauptverantwortung für die Produktgestaltung liegt in der Entwicklungsabteilung, in der IngenieurInnen und TechnikerInnen beschäftigt sind. Neue Produktideen entstehen im Wesentlichen in dieser Abteilung. Aber auch vom Marketing eingebrachte Ideen betreffen in erster Linie technische Produkteigenschaften. Die Dominanz der Technik resultiert daraus, dass die AEG auf eine Tradition von technischen ökologischen Innovationen verweisen kann und dass die technischen Aspekte von Produkten darunter auch ökologisch relevante wie Materialeinsatz oder Rezyklierbarkeit - in der Produktgestaltung umfassend betrachtet werden. Auch die umweltrelevanten Werknormen betrachten hauptsächlich technische Produkteigenschaften.

Neben den positiven Auswirkungen führt der technische Schwerpunkt dazu, dass die ökologischen Aspekte des erweiterten Produktes bislang kaum betrachtet werden. Darunter sind nach Türck alle Vorteile zu verstehen, die im Zusammenhang mit einem Produkt stehen wie Instandhaltung, Service, Garantie, Installation und ähnliche begleitende Kundendienste des anbietenden Unternehmens (3). Ähnliches gilt für Konzepte zur Nutzungsdauerverlängerung oder Nutzungsintensivierung, da beides sich 
nicht technisch realisieren lässt. Die wesentlichen Akteure hierfür wären der Vertrieb und das Marketing; sie sollten daher bereits in der Grundlagenentwicklung in den Gestaltungsprozess eingebunden werden. Da in der AEG Grundlagenentwicklung nur technische Innovationen bearbeitet werden und keine Forschung in bezug auf das erweiterte Produkt oder Vertriebskonzepte stattfindet, sind diese Aspekte schwer in die Entwicklung einzubringen.

Die technische Prägung des Gestaltungsprozesses kann auch als Ursache dafür gesehen werden, dass den frühen, konzeptionellen Phasen weniger Aufmerksamkeit zuteil wird als der späten, technischen Umsetzungsphase.

\section{- Fehlende Verbindung zur Strategieplanung}

Die Produktgestaltung ist nicht systematisch mit der Strategieplanung im Unternehmen verknüpft. Eine unzureichende strategische Orientierung der Produktentwicklung ist nach Bennauer (4) eines der wesentliche Probleme von Produktgestaltung. Strategische Analysen werden nicht explizit für jedes Entwicklungsprojekt durchgeführt. Hierdurch wird proaktiver Umweltschutz erschwert. Durch die Strategieplanung in Kombination mit den Analysen der Initialphase (Kundenanalyse, Konkurrenzanalyse, Umfeldanalyse), können Entwicklungsziele festgelegt werden, die sich an aktuellen und zukünftigen Rahmenbedingungen orientieren. Zusätzlich sollte bereits in dieser Phase eine Auseinandersetzung mit Zielkonflikten erfolgen, die sich aus verschiedenen Anforderungen an Produkte ergeben. Die Verknüpfung mit der Strategieplanung und die Analyse der Rahmenbedingungen könnte auch dazu beitragen, den technischen Schwerpunkt abzuschwächen, da hierdurch andere als technische Ziele identifiziert und festgelegt werden können.

Als ein vielversprechender Ansatz für die Verknüpfung von Strategieplanung und Produktgestaltung wurden Szenarioprozesse identifiziert, die die AEG zu Beginn der 90er für die verschiedenen Produktsparten durchgeführt hat. Szenarioprozesse bilden eine optimale Möglichkeit, aus in die Zukunft gerichteten Umfeldanalysen Folgerungen für die Ausrichtung der Produktentwicklung abzuleiten. Dies wurde bei der AEG jedoch nicht realisiert, da die Ergebnisse der Szenarien nicht in eine langfristige Produkt- oder Strategieplanung eingeflossen sind. Zudem handelte es sich hierbei um ein- malige Prozesse. Für proaktive Umweltmaßnahmen, die eine kontinuierliche Verbesserung erfordern, ist es wichtig, die Rahmenbedingungen regelmäßig zu erfassen und die Ziele daran anzupassen.

\section{Punktuelle Optimierung}

Für die ökologische Produktgestaltung besteht kein integriertes Konzept, sondern viele einzelne Vorschriften. Die Vielzahl der Vorschriften ist ein Zeichen dafür, dass die AEG ökologischer Produktgestaltung große Bedeutung beimisst. Gleichzeitig zeigt sie jedoch, dass an vielen verschiedenen Einzelstellen angesetzt wird, anstatt den Produktgestaltungsprozess umfassend ökologisch zu ergänzen oder umzugestalten. Dies führt dazu, dass ökologische Fragestellungen nicht in allen Entwicklungsphasen konsequent bearbeitet werden, sondern vor allem die Umsetzungsphase optimiert wird.

Das Fehlen eines integrierten Konzeptes macht sich auch in den berücksichtigten Produkteigenschaften bemerkbar: die technischen Eigenschaften werden ökologisch optimiert, nicht jedoch die Eigenschaften des erweiterten Produktes.

\section{- Umweltmanagement zu wenig beteiligt}

VertreterInnen des Umweltmanagements sind nicht in den Produktgestaltungsprozess eingebunden, sondern hauptsächlich für die ökologische Ausrichtung der Produktion sowie für das Rücknahme- und Entsorgungskonzept der Produkte zuständig. Damit nimmt das Umweltmanagement in Bezug auf die Produktgestaltung eine nachsorgende Rolle ein. Die deutliche Trennung zwischen betrieblichem Umweltschutz und Produktgestaltung behindert die Realisierung ganzheitlicher Produktgestaltung. So lange das Umweltmanagement als vom Produktgestaltungsprozess unabhängiger Akteur angesehen wird und demzufolge der Herstellungsprozess und die Entsorgung(skonzepte) der Produkte nicht als Bestandteil der Produktgestaltung gilt, wird eine ökologischen Optimierung entlang der gesamten Produktlinie erschwert. Produkt- und Prozessdesign werden hierdurch in zwei verschiedene Funktionen getrennt. Diese Trennung wird durch die EG-Öko-Audit-Verordnung (EMAS) unterstützt, da nach EMAS die ökologische Optimierung von Produkten nicht Bestandteil des Umweltmanagements ist.

Keoleian/ Menerey (5) beschreiben es hingegen als Ziel umweltgerechten Designs (Life Cycle
Design), Produkt- und Prozessdesign in einer Funktion zusammenzuführen. Ökologische Produktgestaltung kann nur in Verbindung mit betrieblichem Umweltschutz und umweltgerechten Herstellungsverfahren realisiert werden. Die organisatorische Einbindung von VertreterInnen des Umweltmanagements in den Gestaltungsprozess wäre Ausdruck dieser Verbindung. Ein Ansatz zur Einbindung des Umweltmanagements in die Produktgestaltung zeigt sich bei der AEG in vierteljährlichen Entwicklungsgesprächen. Es ist jedoch fraglich, ob diese eine kontinuierliche Einbindung in das Gestaltungsteam ersetzen können.

\section{$>$ Fazit}

Die Analyse des Fallbeispiels verdeutlicht, dass es im Unternehmen bereits vielfältige Ansätze zur ökologischen Produktgestaltung gibt, die zu erfolgreichen ökologischen Produktinnovationen geführt haben. Ökologische Optimierungspotenziale bestehen hier in erster Linie in einer intensiveren Beteiligung weiterer Funktionsbereiche sowie einer systematischeren Einbeziehung ökologischer Fragestellungen insbesondere in die frühen Produktgestaltungsphasen.

\section{Anmerkungen}

(1) Die Ergebnisse der Untersuchung sind ausführlich dargestellt in: Hoffmann, Esther: Ökologische Optimierung der Produktgestaltung, Exemplarische Untersuchung in einem Unternehmen der Elektroindustrie, Berlin 1999: Verlag für Wissenschaft und Forschung.

(2) AEG Hausgeräte GmbH: AEG Grünbuch 1998, S. 90.

(3) Vgl. Türck, Rainer: Das ökologische Produkt, Eigenschaften, Erfassung und wettbewerbsstrategische Umsetzung ökologischer Produkte, Ludwigsburg 1990: Wissenschaft und Praxis.

(4) Bennauer, Ute: Ökologieorientierte Produktentwicklung, Eine strategisch-technologische Betrachtung der betriebswirtschaftlichen Rahmenbedingungen, Heidelberg 1994,

S. 143.

(5) Keoleian, Gregory A./ Menerey, Dan: Sustainable Development by Design: Review of Life Cycle Design and Related Approaches. In: AIR \& WASTE, Vol. 44 (May 1994),

S. 650 .

\section{Die Autorin}

Esther Hoffmann ist wissenschaftliche Mitarbeiterin im Forschungsfeld Ökologische Unternehmenspolitik des IÖW.

Kontakt: IÖW, Potsdamer Str. 105, 10785 Berlin. Tel. 030/ 884594-22, Fax 030/ 8825439,

E-mail: Esther.Hoffmann@ioew.de 
(c) 20I0 Authors; licensee IÖW and oekom verlag. This is an article distributed under the terms of the Creative Commons Attribution Non-Commercial No Derivates License (http://creativecommons.org/licenses/by-nc-nd/3.o/), which permits unrestricted use, distribution, and reproduction in any medium, provided the original work is properly cited. 